\section{P717 USING ETHNOGRAPHY FOR PLANNING AND IMPROVING THE QUALITY OF HIV PREVENTION INTERVENTIONS FOR FEMALE SEX WORKERS IN NIGERIA}

'Baba Mari, 'Kalada Green*, ${ }^{2}$ Shajy Isac, 'Janet Halliday, 'Judith Ariri-Edafe, ${ }^{3}$ Kayode Ogungbemi, ${ }^{4}$ Robert Lorway, ${ }^{5}$ James Blanchard. ${ }^{1}$ Centre for Global Public Health Nigeria, Abuja, Nigeria; ${ }^{2}$ Centre for Global Public Health, University of Manitoba, Winnipeg, Canada; ${ }^{3}$ National Agency for the Control of AIDS, Abuja, Nigeria; ${ }^{4}$ Center for Global Public Health, Department of Community Health Sciences, University of Manitoba, Winnipeg, Canada; ${ }^{5}$ University of Manitoba, Centre for Global Public Health, Department of Community Health Sciences, Winnipeg, Canada

10.1136/sextrans-2019-sti.781

Background Female Sex Workers (FSW) and their clients account for half of HIV infections among key populations $(\mathrm{KP})$ in Nigeria. The aim of this study was to better understand sexual behavior patterns and influences related to HIV vulnerability among men and women in venues that facilitates sexual networks in Nigeria as it relates to sex work.

Methods A qualitative approach was employed using participant observations and face-to-face in-depth key informant interviews. Community ethnographers collected information from FSWs, clients of FSWs and key venue staff at venues where FSWs solicited clients. A semi-structured tool guided the interviews and questions explored how connections occur for the sale of sex and probed the power dynamics within connections.

Results In all, 25 women and 25 men were interviewed. The in-depth interviews describe bar staff as vital players in the sex trade who provide financial and security services for FSWs. In exchange for these services FSWs are expected to pay with cash, commodities or sex. Majority of FSWs experienced violence from clients and law enforcement agents. Interviews indicate that there was a lack of governmental or nongovernmental organizations involved in addressing cases of violence. Participants reported low condom use with intimate partners compared to paying clients. Top on the list of the problems associated with condom use was condom breakage, which was attributed to dryness, lack of lubricants and lengthy duration of sex. In addition, both male and female participants had power over decision making on condom use. Generally, as noted in transcripts, interactions between FSWs and clients switch between commercial sexual relationships and transactional liaisons.

Conclusion The study provides insight into the structure and operational dynamics occurring at venues facilitating sexual networks. Simultaneously exposing the barriers and challenges to HIV prevention service delivery. A combination of strategies-behavioural, biomedical, structural interventions are required in addressing the needs of FSWs.

Disclosure No significant relationships.

\section{P718 \\ RISK AND PLACE: THE ASSOCIATION BETWEEN HOTSPOT TYPOLOGY AND DETERMINANTS OF HIV RISK AMONG FEMALE SEX WORKERS IN UKRAINE}

${ }^{1}$ Eve Cheuk, 'Stella Leung, ${ }^{2}$ Olga Balakireva, ${ }^{3}$ Daryna Pavlova, 'Leigh Mcclarty, ${ }^{4}$ Shajy Isac, ${ }^{1}$ Michael Pickles, ${ }^{5}$ Sharmistha Mishra, ${ }^{6}$ Evelyn Forget, ${ }^{1}$ Robert Lorway, ${ }^{7}$ Paul Sandstrom, ${ }^{1}$ James Blanchard, 'Marissa Becker*. 'University of Manitoba, Centre for Global Public Health, Department of Community Health Sciences, Winnipeg, Canada; ${ }^{2}$ Institute for Economics and Forecasting, Ukrainian National Academy of Sciences, Department for Monitoring-based Research of Social and Economic Transformations, Kyiv, Ukraine; ${ }^{3} N G O$ "Ukrainian Institute for Social Research after Oleksandr Yaremenko"," Monitoring and Evaluation of Social Projects, Kyiv, Ukraine; ${ }^{4}$ India Health Action Trust, Delhi, India; ${ }^{5}$ St. Michael's Hospital, Li Ka Shing Knowledge Institute, Toronto, Canada; ${ }^{6}$ University of Manitoba, Department of Community Health Sciences, Winnipeg, Canada; ${ }^{7}$ Public Health Agency of Canada, National HIV and Retrovirology Laboratories, JC Wilt Infectious Diseases Research Centre, Winnipeg, Canada

\subsection{6/sextrans-2019-sti.782}

Background The environmental contexts and interactions between people within that environment impact a female sex worker's (FSW) individual behaviour and likelihood of HIV acquisition. Understanding how the environment shapes HIV risk can provide important information for HIV prevention programs that go beyond behaviour-based interventions and address more distal factors contributing to HIV risk among FSWs.

Methods A cross-sectional survey was conducted among FSWs in Dnipro, Ukraine between September 2017 and January 2018. 560 FSWs aged $\geq 14$ years were recruited from "hotspots" (locations where FSWs meet clients). We compared determinants of HIV risk between six hotspot typologies (e.g., brothels, home-based, massage parlours, bars/restaurants, parks, highways) using chi-squared tests.

Results Age at start of sex work $(\mathrm{p}<0.001)$, client volume $(p<0.001)$, and HIV testing in the past year $(p<0.001)$ all differed significantly across hotspot typologies. Mean age at start of sex work was oldest for FSWs recruited from highways (24.1 years) and youngest for FSWs recruited from massage parlours (19.8 years). FSWs from highways had the most clients in the past 30 days (mean 22.1 clients) while those from parks had the fewest (mean 17.5 clients). HIV testing in the past year was the most frequent among FSWs from brothels (mean 2.1 times), followed by home-based (1.8 times), massage parlours (1.1 times), highways (1.0 time), bars/restaurants (0.6 times) and parks (0.4 times). The prevalence of physical and sexual violence perpetrated by clients in the past 3 months was $4.3 \%$ and $1.6 \%$ overall. Compared to women from other hotspot typologies, the proportion of FSWs from highways who reported experience of physical and sexual violence by clients were highest $(26.7 \%$ and 10.0\%).

Conclusion Understanding the association between HIV risk factors and hotspot typology can help HIV prevention programs tailor interventions, and target linkage and delivery of services to the relevant subgroups of a key population.

Disclosure No significant relationships. 